\title{
Mechanism of aflatoxin biosynthesis
}

${ }^{1}$ Yabe, K., ${ }^{1,3}$ Wen, Y., ${ }^{1}$ Cai, J., ${ }^{1,4}$ Zeng, H., ${ }^{2}$ Sakuno, E. and ${ }^{2}$ Nakajima, H.

1 National Food Research Institute, Tsukuba

(Ibaraki 305-8642, Japan)

2 Faculty of Agriculture, Tottori University

(Koyama, Tottori 680-8553, Japan)

3 Department of Microbiology, College of Biological Sciences, China Agricultural University

(Beijing 100094, China)

4 Institute of Plant Protection, Chinese Academy of Agricultural Sciences

(Beijing 100094, China)

\begin{abstract}
Aflatoxins are highly toxic and carcinogenic substances mainly produced by Aspergillus flavus and Aspergillus parasiticus. Contamination of agricultural commodities with aflatoxins is a very serious problem all over the world. Aflatoxin biosynthesis has been extensively studied, and now it is known that at least 18 enzyme steps are involved in the pathway from acetyl CoA to aflatoxins. Many genes encoding the enzymes and the transcription factors have been isolated, and those functions have been studied. In the genomes of $A$. parasiticus and A. flavus, at least 25 genes related to aflatoxin biosynthesis make a 70-kb gene cluster. We have investigated many enzyme reactions involved in aflatoxin biosynthesis by using cell-free systems. Our biochemical studies showed the aflatoxin biosynthetic pathway has many unique characters as a secondary metabolism.
\end{abstract}

Key words: aflatoxin biosynthesis, enzyme reactions, OAVN cyclase

Aflatoxins are most potent carcinogenic and toxic substances mainly produced by Aspergillus flavus and $A$. parasiticus ${ }^{1)}$. Contamination of agricultural commodities with aflatoxins has been reported in many agricultural commodities, such as corn, peanuts, and cereals, and in livestock products due to consumption of aflatoxin-contaminated feed by domestic animals. Sterigmatocystin, a latter precursor in aflatoxin biosynthesis, is also toxic and carcinogenic intermediate in aflatoxin biosynthesis, though not as potent as the aflatoxins. Sterigmatocystin is produced as a final product by more than 20 Aspergillus spp. Contamination with sterigmatocystin has been also widely detected.

Aflatoxins $B_{1}\left(A F B_{1}\right), B_{2}\left(A_{F B}\right), G_{1}\left(A F G_{1}\right)$, and $G_{2}\left(A_{F} G_{2}\right)$ are major aflatoxins produced by the fungi. Among them, $\mathrm{AFB}_{1}$ is the most toxic and abundantly produced by fungi ${ }^{2,3)}$. Typically, Aspergillus parasiticus produces these 4 aflatoxins; in contrast, $A$. flavus produces only $\mathrm{B}$-group aflatoxins, $\mathrm{AFB}_{1}$ and $\mathrm{AFB}_{2}$. It was recently reported that the lack of formation of G-group aflatoxins is due to deletion of the region relating to formation of G-group aflatoxins in the genome of $A$. flavus $^{4}$. Furthermore, many genes encoding the enzymes and the transcription factors have been isolated, and those functions have been 
studied $^{2)}$. In the genomes of $A$. parasiticus and $A$. flavus, at least 25 genes related to aflatoxin biosynthesis make a $70-\mathrm{kb}$ gene cluster. The genes involved in sterigmatocystin biosynthesis also form a $60-\mathrm{kb}$ gene cluster in the genome of $A$. nidulans. Although functions of most of all genes have been confirmed, those of several genes remained to be clarified.

\section{Strategies}

We have studied the biosynthetic pathway for aflatoxins ${ }^{3)}$. We first devised some simple and safe methods as follows:

\section{Aspergillus parasiticus NIAH-26 mutant Aflatoxigenic fungi produce large amounts of} aflatoxins as well as those precursors. Production of these products usually interfered with detection of slight amounts of the reaction products in cell-free systems due to background of the endogenous substances. We isolated many mutants from $A$. parasiticus with impaired aflatoxin productivity using the UV photography methods. Some mutants newly accumulated some novel intermediates. Another mutant, NIAH-26, produced neither aflatoxins nor precursors ${ }^{5,6)}$. Since this mutant could produce all enzymes in the pathway from norsolorinic acid (NA) to aflatoxins without any production of aflatoxins, we could safely prepare most of all enzymes involved in aflatoxin biosynthesis.

Feeding experiments The NIAH-26 mutant could convert many precursors to aflatoxins, when each of them was added to the culture medium and then cultured. Since this mutant did not endogenously produce any aflatoxins, aflatoxins newly produced could be sensitively detected. In the feeding experiment, we used a small-scale tip culture method using a 1-ml Pipetman tip as a culture vessel ${ }^{6,7)}$. We could finally find many novel precursors of aflatoxins. Enzyme assays The cell extract, microsome fraction, and cytosol fraction were safely prepared from mycelia of the NIAH-26 mutant. The resulting fractions could be used as the cell-free systems without any purification procedure because they did not contain any endogenous aflatoxins or precursors.

\section{Enzyme reactions involved in aflatoxin biosynthesis}

At least 18 enzyme reactions are involved in the conversion of acetyl-CoA to aflatoxins. Although several side-pathways are also involved, the main pathway for aflatoxin $B_{1}$ production is: acetyl-CoA $\rightarrow$ norsolorinic acid (NA) $\rightarrow$ averantin (AVN) $\rightarrow$ 5'-hydroxyaverantin (HAVN) $\rightarrow$ 5'-oxoaverantin (OAVN) $\rightarrow$ hydroxyversicolorone (HVN) $\rightarrow$ averufin $(A V R) \rightarrow$ versiconal hemiacetal acetate $(\mathrm{VHA}) \rightarrow$ versiconal $(\mathrm{VHOH}) \rightarrow$ versicolorin $\mathrm{B}(\mathrm{VB}) \rightarrow$ versicolorin $\mathrm{A}(\mathrm{VA}) \rightarrow$ demethylsterigmatocystin (DMST) $\rightarrow$ sterigmatocystin $\quad(\mathrm{ST}) \quad \rightarrow \quad O$-methylsterigmatocystin 11-hydroxy- $O$-methylsterigmatocystin $(\mathrm{HOMST}) \rightarrow$ aflatoxin $\mathrm{B}_{1}\left(\mathrm{AFB}_{1}\right)^{3)}$.

(OMST) $\rightarrow$

We have investigated many enzyme reactions involved in aflatoxin biosynthesis by using cell-free systems. We for the first time reported that 5'-hydroxyaverantin (HAVN) and 5 '-oxoaverantin (OAVN) were precursors of aflatoxins ${ }^{8)}$. Feeding experiment of the NIAH-26 mutant with either substance caused production of aflatoxins. Incubation of averantin (AVN) with the microsome fraction of this mutant produced HAVN, which was converted to OAVN and then to averufin (AVR). The flow of the reactions were clearly detected using thin layer chromatography. We could clarify most of all enzyme steps in aflatoxin biosynthesis.

One enzyme is involved in two different reactions in aflatoxin biosynthesis

We recently purified OAVN cyclase that catalyzes the reaction form OAVN to AVR in $A$. parasiticus $^{8}{ }^{8}$. The purified OAVN cyclase enzyme catalyzed two reactions, the reaction from $\mathrm{OAVN}$ to $\mathrm{AVR}$ and the reaction from $\mathrm{VHOH}$ to $\mathrm{VB}^{9}$. Interestingly, the $\mathrm{N}$-terminal 
25-amino-acid sequence of the OAVN cyclase completely matched an internal sequence of the versiconal $(\mathrm{VHOH})$ cyclase that was deduced from its gene $(v b s)$. To determine the relationship between the enzyme activities of OAVN cyclase and VHOH cyclase in the cells, we performed a competition experiment between OAVN and $\mathrm{VHOH}$ using the cytosol fraction of $A$. parasiticus NIAH-26. AVR was produced from OAVN by the cytosol fraction. However, when VHOH was added to the same reaction mixture, VB was newly formed: in contrast, the formation of AVR from OAVN remarkably decreased. These results suggested that OAVN and VHOH compete for the same enzyme in the cytosol (Fig. 1).

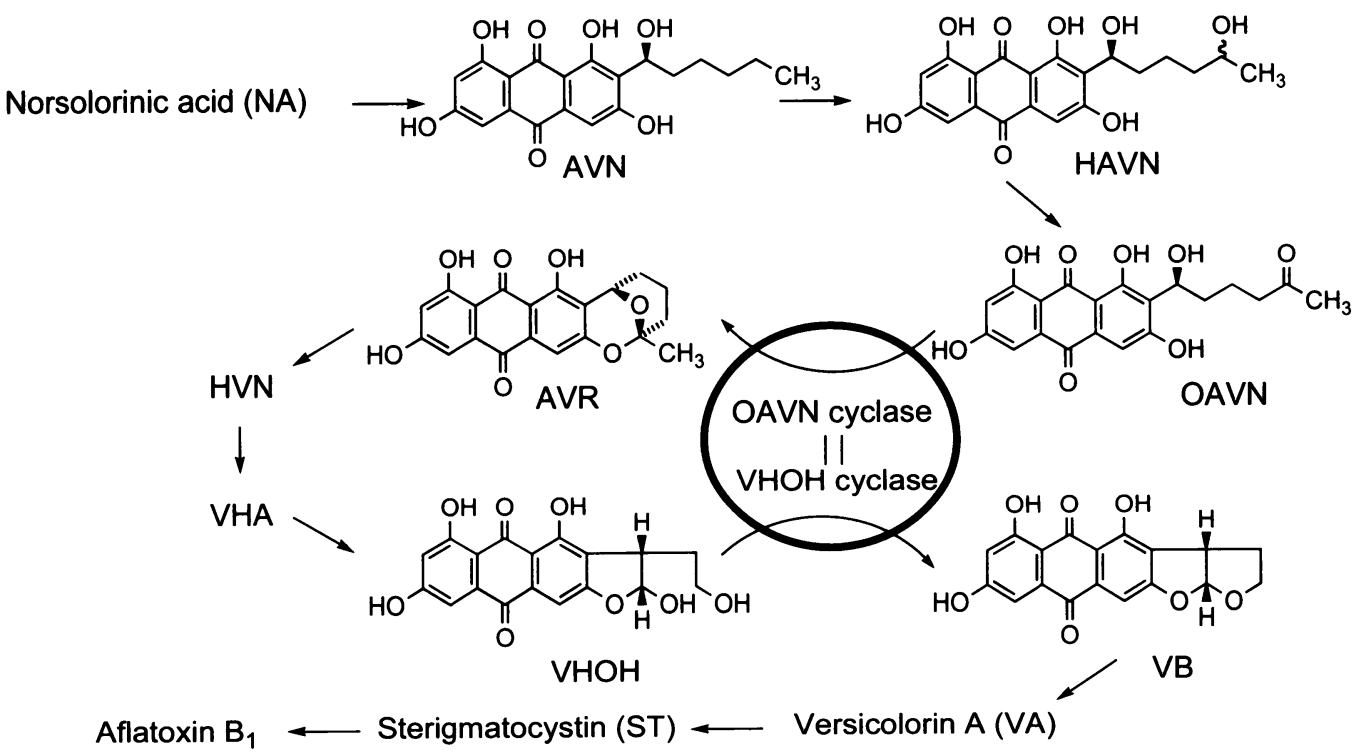

Fig. 1. The same enzyme works as OAVN cyclase as well as VHOH cyclase.

The recombinant Vbs protein was then expressed in Pichia pastoris expression system. When the cell-extract of the transformed yeast was incubated with OAVN, AVR was newly formed. In contrast, when $\mathrm{VHOH}$ was added to the same system instead of OAVN, VB was newly formed. These results demonstrated that the $v b s$ gene is involved in both reactions from OAVN to AVR and from VHOH to VB.

The $v b s$ gene is a sole gene encoding a cyclase (dehydrase) in the aflatoxin gene cluster. The $v b s$ gene had been isolated to encode the latter VHOH cyclase enzyme in the pathway. If the same enzyme is also involved in the early OAVN cyclase step, disruption of the $v b s$ gene may primarily cause inhibition of the former reaction, causing accumulation of the substrate of the early enzyme, that is, OAVN. We did disruption of the $v b s$ gene, and pigments accumulated in the resulting $v b s$-deletion mutant were analyzed. The same mutant accumulated five kinds of orange pigments, which corresponded to OAVN, HAVN, AVN and so on. These results indicated that the cyclase encoded by the $v b s$ gene is also involved in the reaction from OAVN to AVR in aflatoxin biosynthesis.

One enzyme for one step is common in many metabolic pathways. This was the first report that the same enzyme was involved in two different steps in the same pathway. It is interesting from an evolutionary standpoint. Long time ago these two steps in aflatoxin biosynthesis were likely catalyzed by two different enzymes, one of which was a product of $v b s$ gene. During evolution, one of them was probably deleted, but this did not affect aflatoxins production because the product of the remaining $v b s$ gene could catalyze both reactions. The product of the remaining $v b s$ gene might be necessary for fungal survival. The 
function and evolution of secondary metabolism remain to be studied.

\section{Overview}

Our biochemical studies showed that both soluble and membrane enzymes are intermittently involved in the pathway; at least four membrane enzymes and more than seven soluble enzymes are related. Also, this pathway appeared to be a high energy-consuming one because most of all steps require various cofactors such as NADPH and $S$-adenosylmethionin for their activities. The supply of the cofactors from primary metabolism may be a key factor regulating this secondary metabolism ${ }^{3)}$.

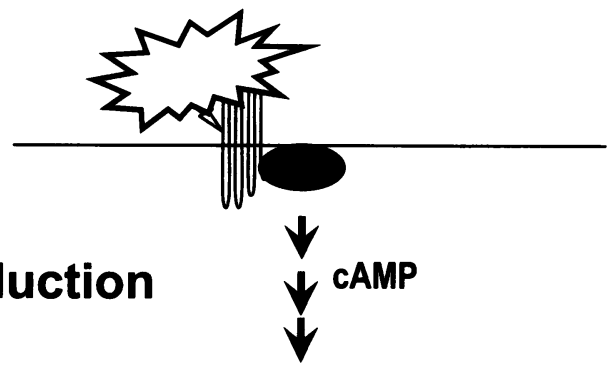

Signal transduction

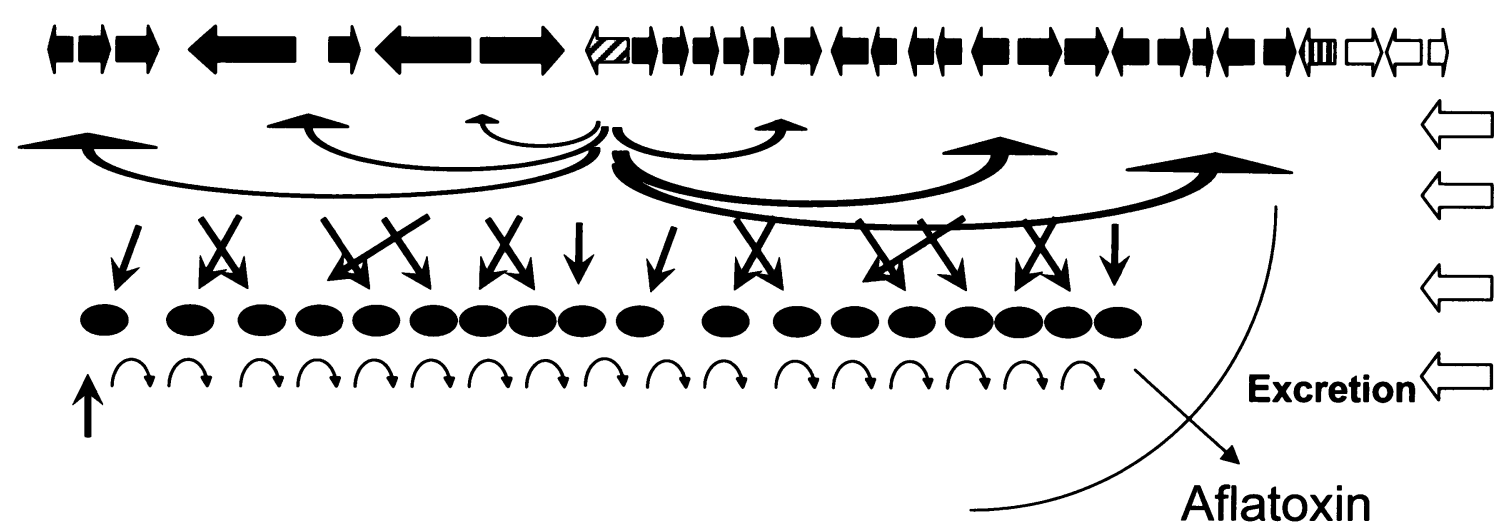

Fig. 2. Model for control of aflatoxin production by culture conditions. Open arrows show possible targets for prevention of aflatoxin production.

Culture conditions affect secondary metabolisms. Some factors do not enter the cell, but bind to a certain receptor at the cell surface and initiate the transmission of a signal into the cell interior. This "signal transduction" is one of central themes in life science. Since aflatoxin productivity is affected by many factors such as carbon sources, temperature, and so on, the similar mechanism has been suggested in aflatoxin production (Fig. 2). Involvements of G-protein and cAMP in aflatoxin biosynthesis have been reported. Expression of all genes in the aflatoxin gene cluster is regulated by the positive regulatory gene, aflR. The expression of the aflR gene is induced by the signal transduction. Therefore, every step involved in the flow from the outside signal to aflatoxin production can be used as a target for inhibition. We may be near to the development of practical methods for preventing aflatoxin contamination. 


\section{References}

1) Payne, G.A., and Brown, M.P.: Annu. Rev. Phytopathol., 36, 329-362 (1998)

2) Yu, J., Chang, P.-K., Ehrlich, K.C., Cary, J.W., Bhatnagar, D., Cleveland, T.E., Payne, G.A., Linz, J.E., Woloshuk, C.P., Bennett, J.W.: Appl. Environ. Microbiol., 70, 1253-1262 (2004)

3) Yabe, K., and Nakajima, H.: Appl. Microbiol. Biotechnol., 64, 745-755 (2004)

4) Ehrlich, K.C., Chang, P.-K., Yu, J., Cotty, P.J.: Appl. Environ. Microbiol., 70, 6518-6524 (2004)

5) Yabe, K.: Mycotoxins, 53, 103-111 (2003)

6) Yabe, K., Nakamura, H., Ando, Y., Terakado, N., Nakajima, H., Hamasaki, T.: Appl. Environ. Microbiol., 54, 2096-2100 (1988)

7) Yabe, K., Ando, Y., Hamasaki, T.: Appl. Environ. Microbiol., 54, 2101-2106 (1988)

8) Sakuno, E., Yabe, K., and Nakajima, H.: Appl. Environ. Microbiol., 69, 6418-6426 (2003)

9) Sakuno, E., Wen, Y., Hatabayashi, H., Arai, H., Aoki, C., Yabe, K., Nakajima, H.: Appl. Environ. Microbiol., 71, 2999-3006 (2005) 